\title{
СИНТЕЗ СИСТЕМЫ АВТОМАТИЧЕСКОГО УПРАВЛЕНИЯ РОБОТОТЕХНИЧЕСКИМ УСТРОЙСТВОМ ДЛЯ ПРИГОТОВЛЕНИЯ ПОЛУФАБРИКАТОВ ПЕЛЬМЕННОЙ ПРОДУКЦИИ ОСОБЫХ ФОРМ
}

\author{
Голубков П. ${ }^{1}$, Путников Д. ${ }^{2}$, Егоров В. ${ }^{3}$, Похлебина Н. ${ }^{4}$, Габуєв К. ${ }^{5}$, Гонгало В. ${ }^{6}$ \\ 1,2,3,4,5,6 - Одесская национальная академия пищевых технологий, Одесса, Украина \\ ORCID: ${ }^{1} 0000-0002-7663-6772 ;{ }^{2} 0000-0003-2577-8858 ;{ }^{3} 0000-0003-4699-834 X ;{ }^{4} 0000-0002-9789-608 X,{ }^{5} 0000-0003-0704-$ \\ 9774; ${ }^{6} 0000-0002-5392-6574$. \\ E-mail: ${ }^{1}$ pavelsergeevichgolubkov@gmail.com, ${ }^{2}$ ulkiorrrra@gmail.com, ${ }^{3}$ mechatronics.robotlab@gmail.com, \\ ${ }^{4}$ nadin_design@ukr.net, ${ }^{5}$ kostyacart@gmail.com, 호kipper9629@gmail.com.
}

Copyright (C) 2018 by author and the journal “Automation technologies and business - processes. This work is licensed under the Creative Commons Attribution International License (CC BY). http://creativecommons.org/licanses/by/4.0

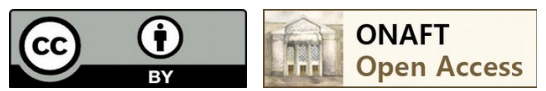

DOI: $10.15673 /$ atbp.v10i4.1231

Аннотация:В работе рассматривается синтез системы автоматического управления нагревом теста в процессе приготовления пельменной продукции сложной труднореализуемой кубической формы. Так как, в процессе производства продукта необходимо поддерживать заданную положительную температуру теста, находящегося на замороженном фарше, то задача управления технологическим процессом сведена к задаче регулирования температурного режима на нагревательных элементах оборудования. В работе также рассмотрена система автоматической оптимизации заданного значения температуры в зоне слепки пельменной продукции, которая в соответствии с математической моделью дает возможность, используя полученные температурные значения, рассчитать и проверить в реальном времени необходимые для получения выпускаемой продукции, значение температурного режима согласно установленным регламентам. Результаты работы системы произведены в nакете Simulink мощного программного обеспечения Matlab, предназначенного для математического моделирования различных технологических процессов и проверены активными экспериментами, что наглядно иллюстрируется графиками переходных процессов. Оборудование, на котором производились активных эксперименты имеет три зоны подогрева теста изготавливаемой пельменной продукции и две зоны формования. В зонах подогрева система управления производственным процессом получает данные о температуре теста и выдаёт управляющие сигналы на нагревательные элементы. Расчеты производятся в каждой зоне отдельно и параллельно производится расчет математической модели. Имея математические модели САР производит расчеты и сравнительный анализ в режиме реального времени, используя таблицу регламентных значений.

Abstract:It is discussed in this paper the synthesis of the automatic control system for heating the dough in the process of preparing ravioli products of a complex cubic form. Since, during the preparation of the product, it is necessary to maintain the desired positive temperature of the dough, which is on frozen mincemeat, the task of controlling the technological process is defined as the task of regulating the temperature mode on the equipment heaters. It is also discussed in this paper the system for automatically optimizing the temperature setpoint in the zone of ravioli, which, in accordance with the mathematical model, makes it possible, using the obtained temperature values, to calculate and check in real-time the temperature mode according to the established regulations value required for the production. The results of the system produced in the Simulink package of program Matlab that is used for mathematical modelling of various technological processes and verified by active experiments, which are vividly illustrated by transient graphs. The equipment on which the active experiments were carried out has three zones for heating the dough of manufacturing ravioli products and two molding zones. In heating zones, the process control system receives data about the dough temperature and provides control signals to the heating elements. Calculations are made in each zone separately and in parallel the calculation of the mathematical model is made. Having mathematical models SAR performs calculations and comparative analysis in real time using the table of established values.

Ключевые слова: синтез САУ процессом нагрева пельменя кубической формы, САР температуры нагрева теста пельменя, САО температуры нагрева теста пельменя, полуфабрикаты пельменной продукции. 
Key words: synthesis of SAC by ravioli heating process, SAR by ravioli heating temperature, SAO by ravioli heating temperature

Целью работы является синтез системы автоматического управления процессом нагрева пельменной продукции особых форм.

Достижение цели осуществляется при выполнении следующих задач:

1) Синтез многомерной системы автоматического регулирования температуры нагрева теста для производства пельменной продукции в зонах оборудования;

2) Синтез системы автоматической оптимизации температуры нагрева теста пельменной продукции в третьей зоне оборудования, согласно регламентных значений.

Суть работы: В процессе приготовления пельменной продукции тесто с положительной температурой непрерывно находится в контакте с замороженным фаршем. Температура фарша равна температуре заморозки и может быть от $-40^{\circ} \mathrm{C}$ до $-15^{0} \mathrm{C}$. По технологии для приготовления пельменной продукции тесто должно иметь температуру не ниже $23^{\circ} \mathrm{C}$. Однако, из-за того, что тесто находится в контакте с замороженным фаршем оно интенсивно охлаждается, в связи с чем в процессе работы необходимо изменять и поддерживать температуру в окрестностях заданных значений согласно таблицы регламентов. Задача управления процессом нагрева теста содержащегося в производимой пельменной продукции сводится $\mathrm{k}$ задаче регулирования и подержания положительной заданной температуры теста и недопущения нагрева замороженного фарша свыше регламентного значения. Синтез системы автоматического управления начинается с задачи определения регламента регулируемых переменных, результаты которой наглядно иллюстрируют с помощью таблицы регламента управляемых переменных.

Таблица 1 - Таблица регламентов

\begin{tabular}{|c|c|c|c|c|c|c|}
\hline \multirow{3}{*}{$\begin{array}{c}\text { Название } \\
\text { параметров }\end{array}$} & \multirow{3}{*}{$\begin{array}{c}\text { Обозна- } \\
\text { чения }\end{array}$} & \multirow{3}{*}{$\begin{array}{c}\text { Единицы } \\
\text { измерения }\end{array}$} & \multirow{3}{*}{$\begin{array}{c}\text { Номинальное } \\
\text { значение } \\
\text { параметра или } \\
\text { функция } \\
\text { изменения } \\
\text { номинального } \\
\text { значения }\end{array}$} & \multicolumn{3}{|c|}{ Допустимые отклонения от номинала } \\
\hline & & & & \multirow{2}{*}{$\begin{array}{c}\text { Длительные } \\
(\mathrm{t} \rightarrow \infty)\end{array}$} & \multicolumn{2}{|c|}{$\begin{array}{c}\text { Кратковременные } \\
(0<\mathrm{t}<<\infty)\end{array}$} \\
\hline & & & & & величина & время, сек \\
\hline 1 & 2 & 3 & 4 & 5 & 6 & 7 \\
\hline $\begin{array}{l}\text { тесто в } 1 \\
\text { камере }\end{array}$ & $\mathrm{t}_{\mathrm{T} 1}$ & ${ }^{0} \mathrm{C}$ & +25 & \pm 2 & \pm 5 & 60 \\
\hline $\begin{array}{l}\text { тесто в } 2 \\
\text { камере }\end{array}$ & $\mathrm{t}_{\mathrm{T} 2}$ & ${ }^{0} \mathrm{C}$ & +25 & \pm 2 & \pm 5 & 60 \\
\hline $\begin{array}{c}\text { тесто в } 3 \\
\text { камере }\end{array}$ & $\mathrm{t}_{\mathrm{T} 3}$ & ${ }^{0} \mathrm{C}$ & +25 & \pm 2 & \pm 5 & 60 \\
\hline время слепки & $\tau_{\text {сл }}$ & сек & $3-5$ & \pm 2 & \pm 5 & 60 \\
\hline $\begin{array}{c}\text { форма } \\
\text { пельменя }\end{array}$ & Sf & - & 1 & - & - & - \\
\hline
\end{tabular}

На рис. 1 приведена структурная схема системы автоматического управления процессом нагрева теста производимой пельменной продукции.

Схема состоит из двух подсистем - подсистемы автоматического регулирования и подсистемы автоматической оптимизации. Объектом управления в системе автоматического регулирования является температура теста пельменной продукции в трех зонах. Таким образом, ОУ является многомерным и состоит из 4 управляемых

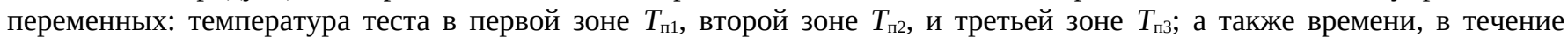

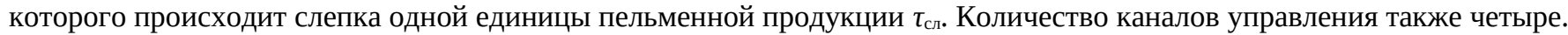
Принципиальной особенностью схемы является то, что регулирование температуры в каждой зоне осуществляется при помощи своего регулятора. Регулирование осуществляется методом непрерывного измерения температуры теста при помощи датчика температуры, расположенного на лапках поршней цилиндров барабана. 


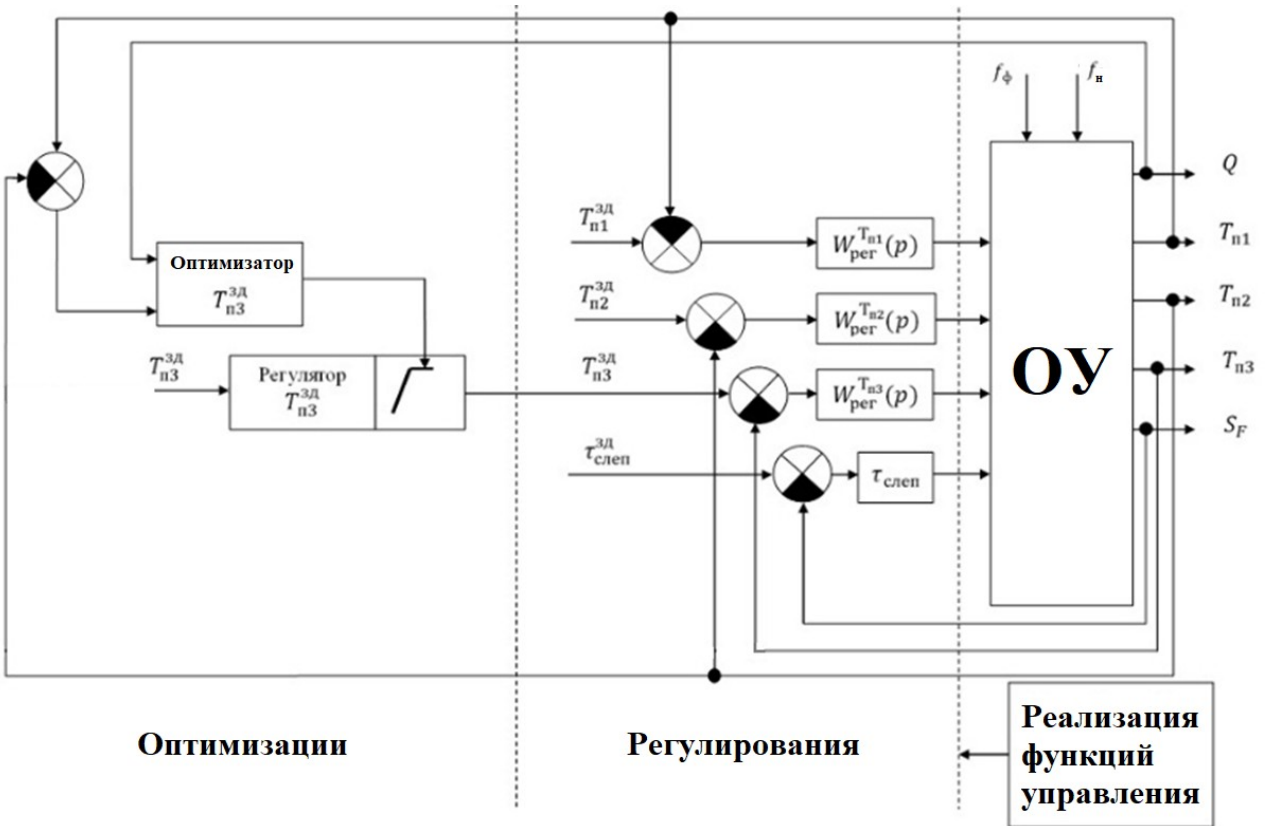

Рис. 1 - Концептуальная структурная схема САУ

Важно подчеркнуть также, что датчик температуры измеряет не температуру самого теста, а температуру на лапках, в которых расположены нагревательные элементы производящие нагрев пельменного продукта. На каждый из информационных каналов датчиков также непрерывно действует возмущающее воздействие в виде отрицательной температуры замороженного фарша, которое приводит к охлаждению теста. Время слепки пельменя, как видно из схемы не регулируется и задано согласно температуры таблицы регламентов. Вторая подсистема реализует функцию оптимизации температурного режима в третьей зоне, посредством использования информации температур в первых двух зонах. Суть ее заключается в изменении заданного значения температуры в третьей камере согласно расчетов, полученных в первых двух камерах для получения пельменной продукции в камере формования. Для решения задачи синтеза системы автоматического управления была построена имитационная модель объекта управления в программе математического моделирования Matlab, а также система управления. Проведены активные эксперименты, подтверждающие работоспособность системы. Структурная схема имитационной модели системы управления приведена на рис. 2.

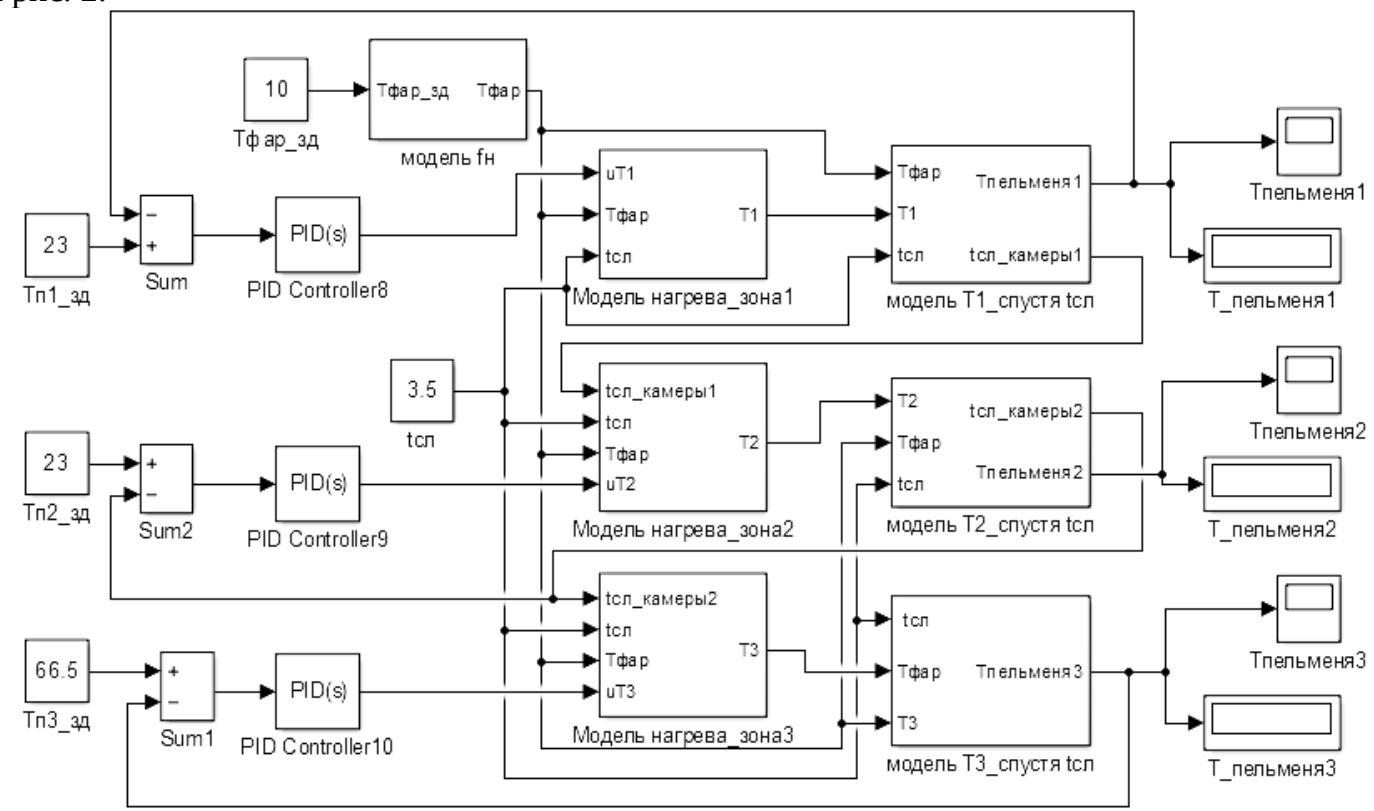

Рис. 2 - Схема моделирования САУ процессом нагрева теста пельменного продукта при автоматическом регулировании температурного режима обработки сырья, вариант регулирования температуры в третьей зоне при фиксированном заданном значении $\theta_{3}^{3 д}=66,5^{\circ} \mathrm{C}$ и времени слепки пельменя $t_{\mathrm{cr}}=1,5 \mathrm{c}$ 

http://www.atbp.onaft.edu.ua/

На рис. 3 приводится внутреннее содержимое блоков.

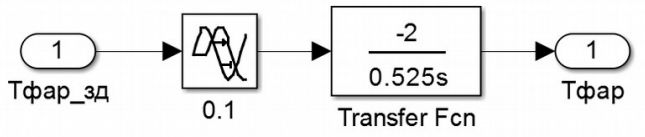

a)

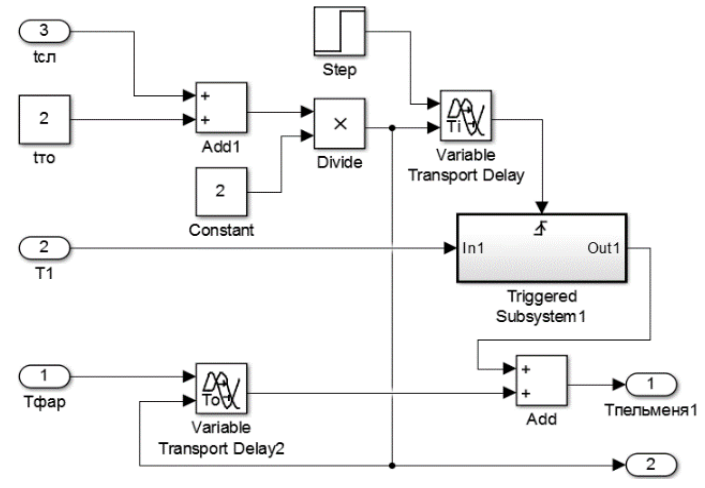

б)

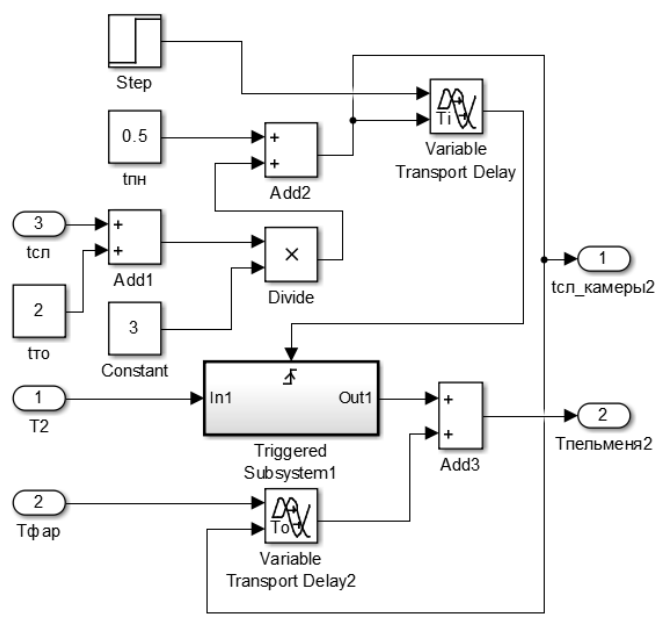

B)

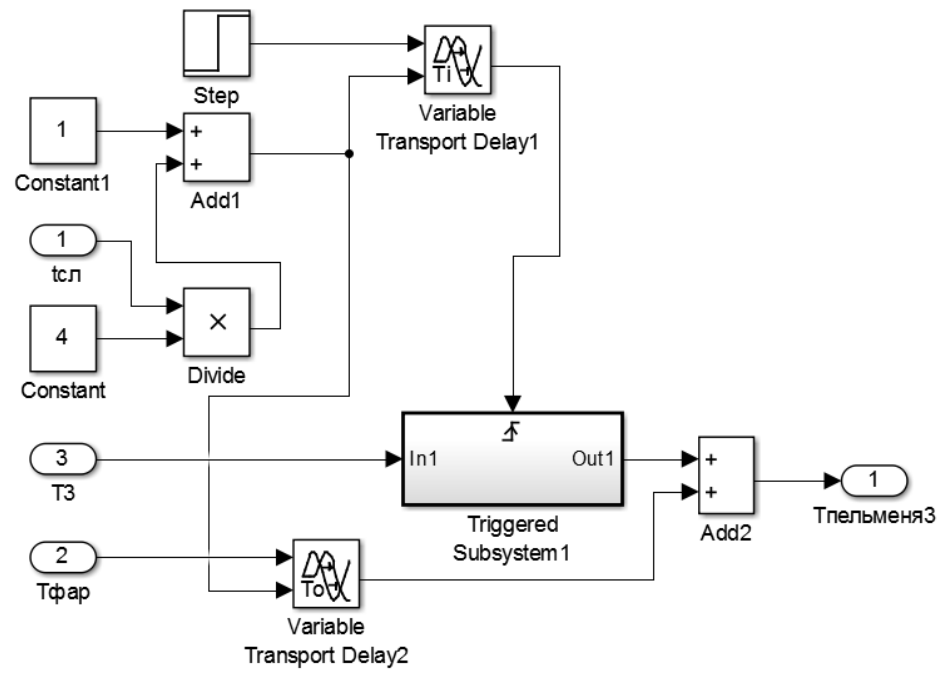

г) 


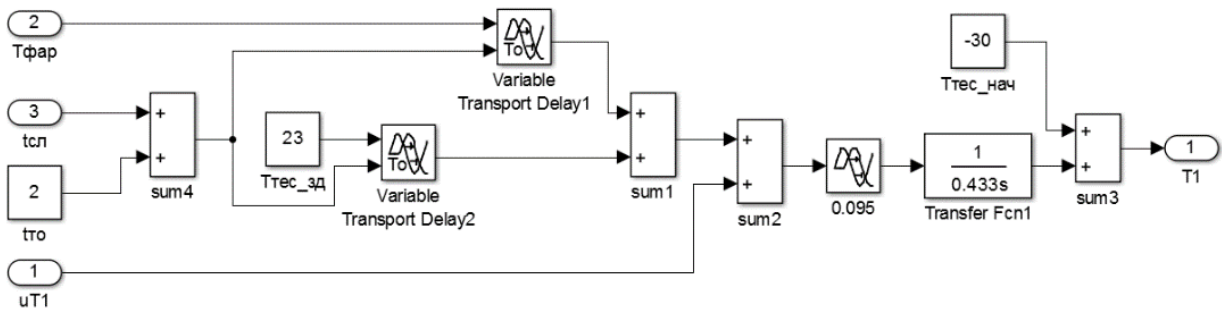

д)

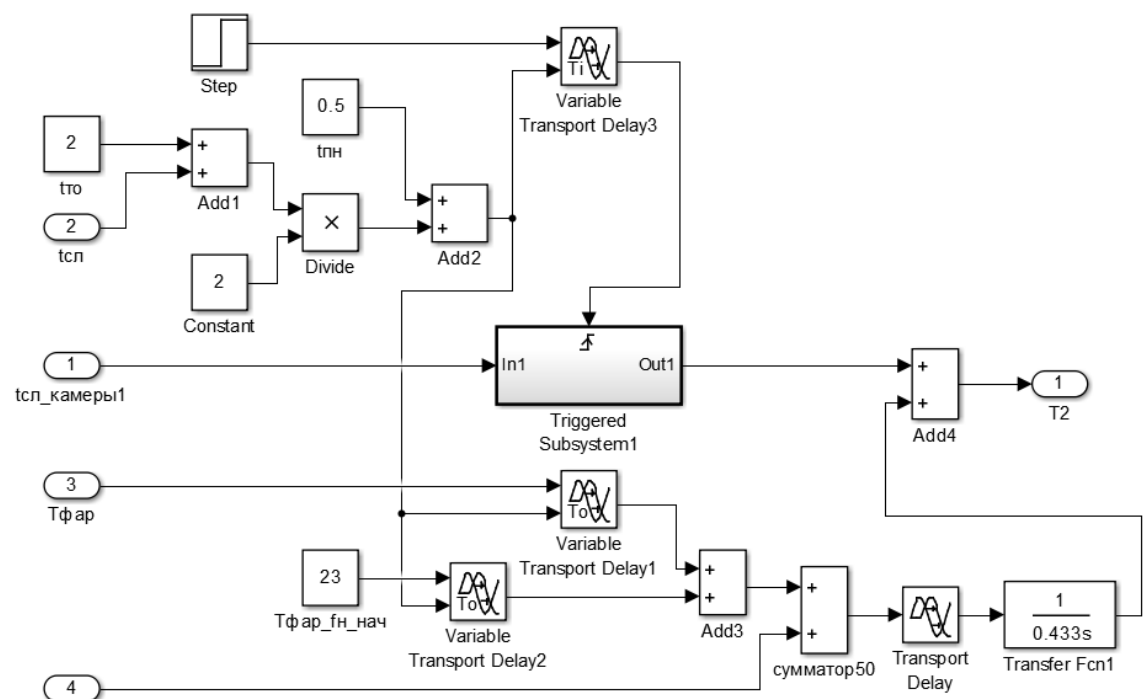

e)

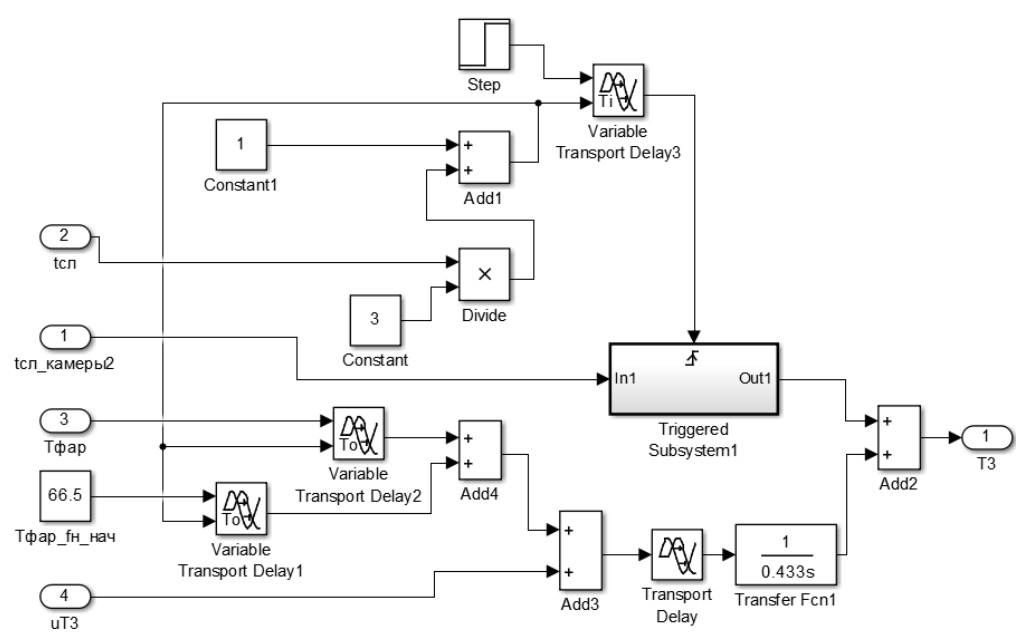

ж)

$a$-модель неконтролируемых возмущений температуры замороженного фарша; б - модель расчета времени задержки при переходе из зоны приема сырья в первую зону нагрева пельменного продукта; в - модель расчета времени задержки при переходе из первой во вторую зону нагрева пельменного продукта; г - модель расчета времени задержки при переходе из второй в третьей зону нагрева пельменного продукта; д - модель нагрева теста пельменного продукта в первой зоне; е - модель нагрева теста пельменного продукта во второй зоне; ж - модель нагрева теста пельменного продукта в третьей зоне пельменного продукта

\section{Рис. 3 - Содержимое блоков структурной схемы САУ}

В качестве устройства управления использовался регулятор с ПИД законом регулирования, дающий на практике удовлетворительные результаты при решении большинства задач управления, что было подтверждено при проведении активных экспериментов. Для параметрического синтеза ПИД регулятора был использован блок PID 
библиотеки Matlab Simulink со встроенным алгоритмом расчета его настроечных параметров. Содержимое блока системы автоматической оптимизации заданного значения температуры третьей камеры показано на рис. 4.

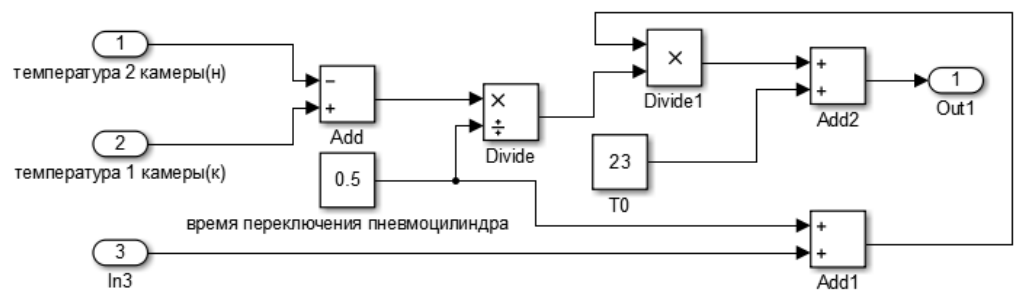

Рис. 4 - Содержимое блока оптимизации заданного значения температуры нагрева теста пельменя в третьей зоне

На рис. 4 приведены результаты моделирования САР имитационной модели с оптимальными настроечными параметрами ПИД-регулятора.

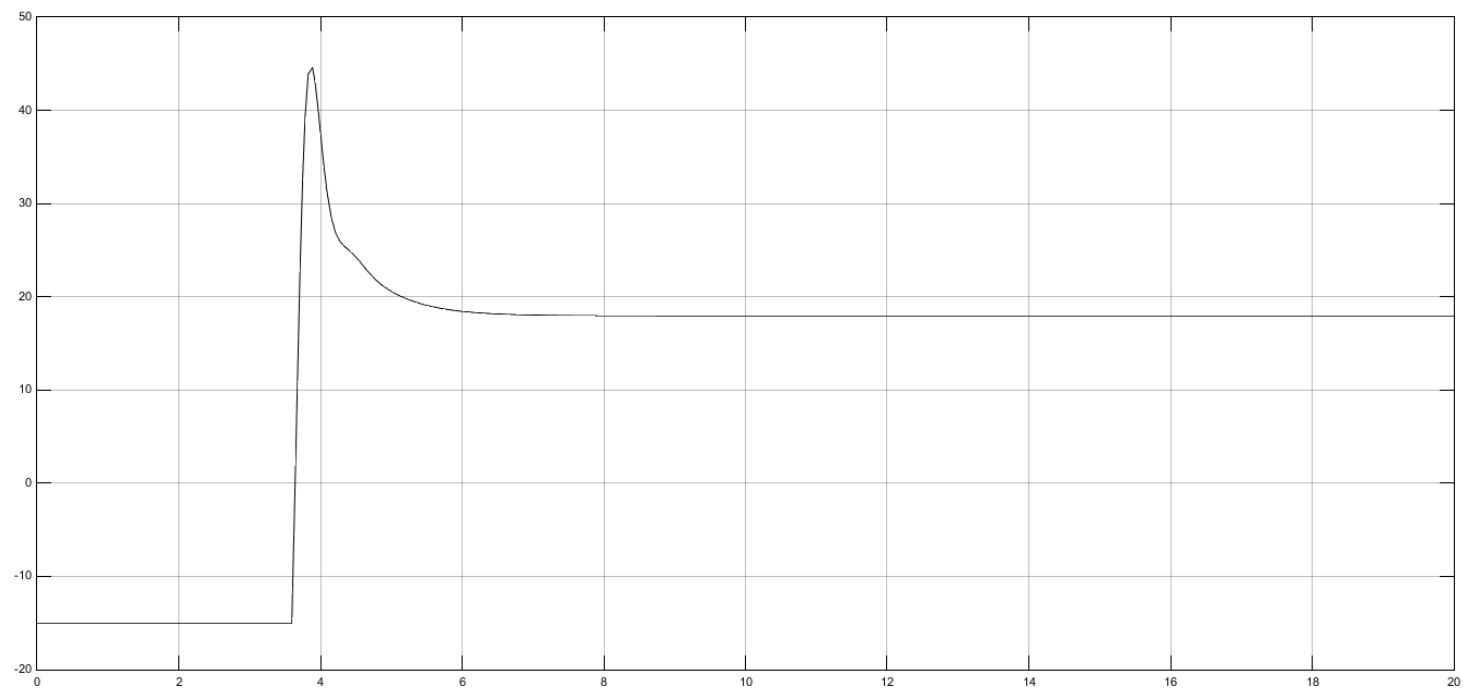

a)

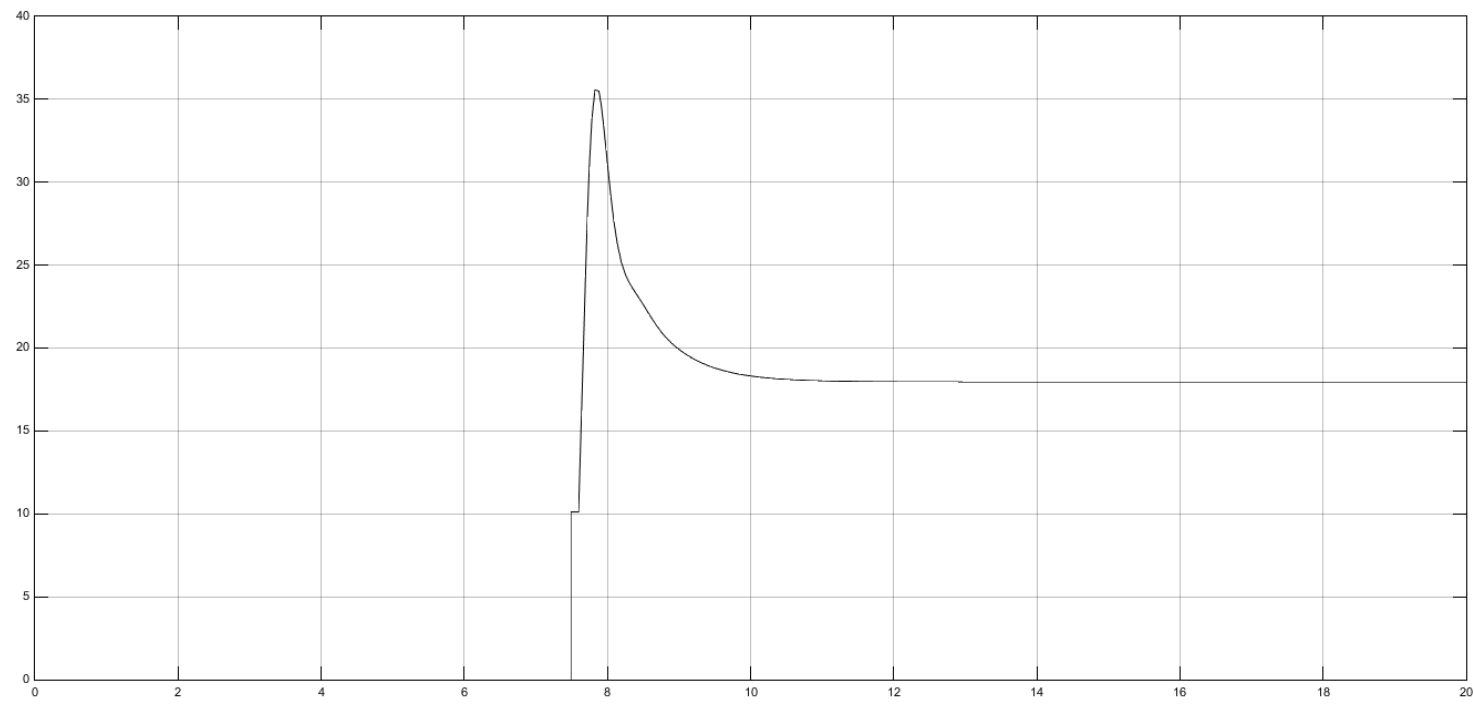

б) 


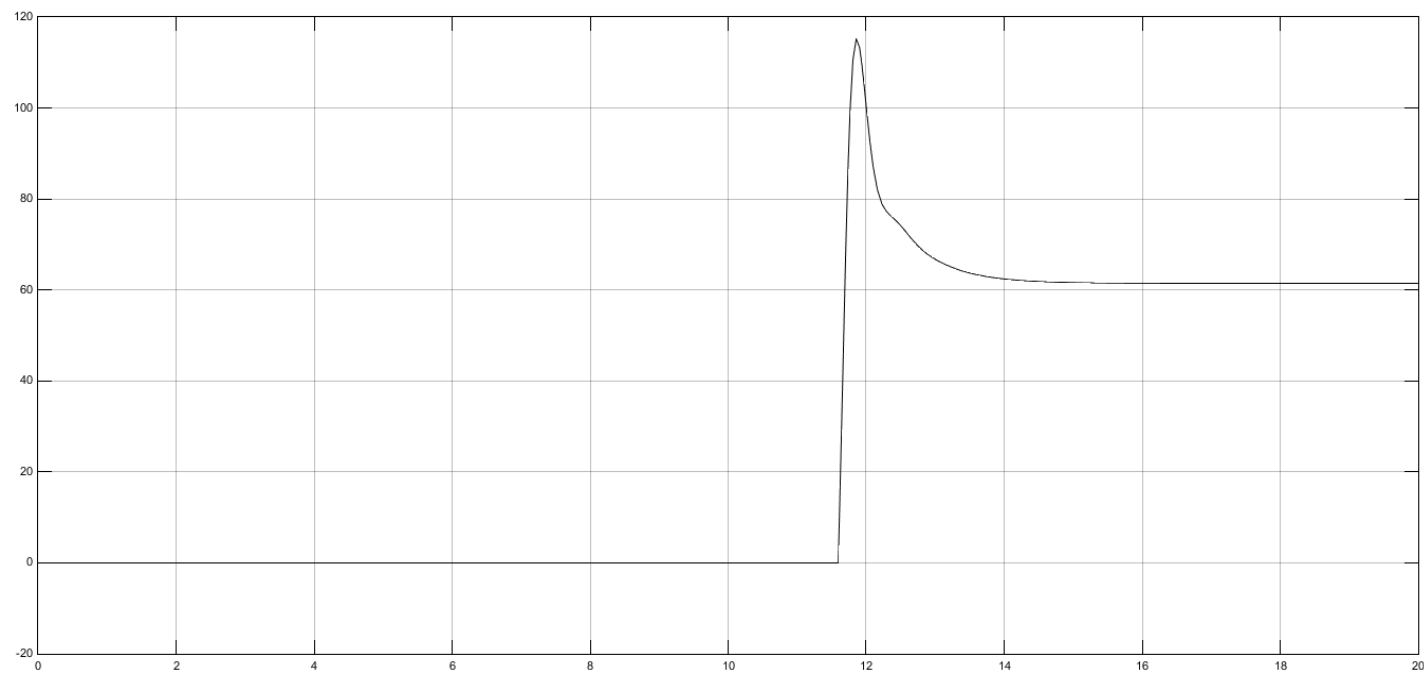

B)

Рис. 5 - Моделирование процесса нагрева теста пельменного продукта при автоматическом регулировании температурного режима обработки сырья для $\theta_{1}^{3, I}=23^{\circ} \mathrm{C}, \theta_{2}^{3,4}=23^{\circ} \mathrm{C}$ и фиксированных значениях заданного значения температуры в третьей зоне $\theta^{\theta^{3,}}=66,5^{\circ} \mathrm{C}$ и времени слепки $t_{\mathrm{cr}}=1,5 \mathrm{c}$

Приведенные на рис. 5 графики моделирования процесса нагрева теста пельменного продукта показывают кратковременные выходы регулируемой переменной за рамки допустимых регламентных значений температур, что видно на рис. 5, в. Несмотря на это, выход температуры является кратковременным и не критичным для производства, т.к. в действительности, по результатам проведенных экспериментов, за такой короткий промежуток времени не будет нарушена структура теста и, следовательно, брака продукции не будет.

\section{Выводы.}

Приготовление пельменной продукции строгой кубической формы является актуальным направлением, так как, во-первых, такая форма позволяет избежать различных подделок со стороны конкурентов, тем самым повысив доверие конечных покупателей к фирме производителю; во-вторых, пельмени такой формы будут занимать все полезное место в упаковке, тем самым, позволяя экономить на упаковочном материале, а также месте при хранении на складах и транспортировке до 20\%. Следовательно, автоматизация процесса приготовления пельменной продукции строгой кубической формы является актуальной задачей.

В результате выполнения поставленных задач на автоматизацию температурного режима обработки пельменной продукции в процессе его приготовления видно, что система автоматического регулирования полностью соответствуют регламенту ведения технологического процесса. Следовательно, можем утверждать об экономической составляющей при хранении, изготовлении, фасовке и транспортировке производимой продукции. Применение системы автоматического управления, полученной в ходе синтеза для работы в технологическом процессе производства полуфабрикатов пельменной продукции кубической формы обуславливает экономическую эффективность и сокращает время производства.

\section{Список использованных источников}

[1] Berry M.R., Bradshaw J.G., Kohnhorst A.L. Heating Characteristics of Ravioli in Brine and in Tomato Sauce Processed in Agitating Retorts // Journal of Food Science. 1985. № 3 (50). C. 815-822;

[2] Morgan M.T., Haley T.A. Design of Food Process Controls Systems 2007. 485-552 c;

[3] Martin С.К. [и др.]. Measuring food intake with digital photography // Journal of Human Nutrition and Dietetics. 2014. № SUPPL.1 (27). C. 72-81;

[4] Yousefi-Darani A. [и др.]. Closed loop control system for dough fermentation based on image processing // Journal of Food Process Engineering. 2018. № 5 (41);

[5] Lima J., Moreira J.F.P., Sousa R.M. Remote supervision of production processes in the food industry $2016.1123-1127$ c;

[6] Kolberg D., Zühlke D. Lean Automation enabled by Industry 4.0 Technologies 2015. 1870-1875 c.

\section{References}

[1] M. R. Berry, J. G. Bradshaw, and A. L. Kohnhorst, "Heating Characteristics of Ravioli in Brine and in Tomato Sauce Processed in Agitating Retorts,” Journal of Food Science, vol. 50, no. 3, pp. 815-822, 1985. 
[2] M. T. Morgan and T. A. Haley, "Design of Food Process Controls Systems,” in Handbook of Farm Dairy and Food Machinery, 2007, pp. 485-552;

[3] C. K. Martin, T. Nicklas, B. Gunturk, J. B. Correa, H. R. Allen, and C. Champagne, "Measuring food intake with digital photography,” J. Hum. Nutr. Diet., vol. 27, no. SUPPL.1, pp. 72-81, 2014;

[4] A. Yousefi-Darani, O. Paquet-Durand, V. Zettel, and B. Hitzmann, "Closed loop control system for dough fermentation based on image processing," J. Food Process Eng., vol. 41, no. 5, 2018;

[5] J. Lima, J. F. P. Moreira, and R. M. Sousa, "Remote supervision of production processes in the food industry,” in IEEE International Conference on Industrial Engineering and Engineering Management, 2016, vol. 2016-January, pp. 11231127 ;

[6] D. Kolberg and D. Zühlke, “Lean Automation enabled by Industry 4.0 Technologies,” in IFAC-PapersOnLine, 2015, vol. 48, no. 3, pp. 1870-1875.

\title{
ИДЕНТИФИКАЦИЯ МОДЕЛЕЙ ИСТЕЧЕНИЯ ЗЕРНА ИЗ ПОДСИЛОСНЫХ ЗАДВИЖЕК ДЛЯ АСОЗ ПТЛ ПЕРЕГРУЗКИ ЗЕРНА
}

\author{
Кирьязов И.Н. ${ }^{1}$, Шестопалов С.В. ${ }^{2}$, Степанов М.Т. ${ }^{3}$, Хобин В.А. ${ }^{4}$ \\ 1,2,3,4 - SE Group International, ОНАПТ (Украина) \\ E-mail: 1ivan.kiryazov@se.ua, ${ }^{2}$ stanislav.shestopalov@se.ua, ${ }^{3}$ stepanov197818@gmail.com, ${ }^{4}$ khobin@onaft.edu.ua \\ Odessa National Academy of Food Technologies, Odessa, Ukraine
}

Copyright (C) 2018 by author and the journal “Automation technologies and business - processes. This work is licensed under the Creative Commons Attribution International License (CC BY). http://creativecommons.org/licanses/by/4.0

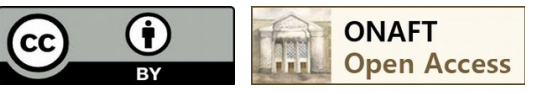

DOI: $10.15673 /$ atbp.v10i4.1232

Аннотация:Компания S-engineering входящая в холдинг SE Group International занимает лидирующие позиции в области автоматизации технологических процессов зерноперерабатывающей отрасли включая в свои проекты инновационные разработки. В частности компания занимается разработкой инновационных систем автоматической оптимизации загрузки поточно-транспортных линий (АСОЗ ПТЛ) зерновых терминалов. Разрабатываемые системы предназначены для повышения производительности ПТЛ, снижения удельных затрат электрической энергии на перегрузку, предотвращения аварийных ситуаций, связанных с завалами зерна в башмаках норий и надвесовом бункере весов из-за перегрузки ПТЛ, Указанные задачи решаются системой в условиях формирования потока зерна одновременно из нескольких источников. ACO3 реализует технологии Leffol \& Senuтас $(L \& S)$, запатентованные SE Group International. Технология LEFFOL: способ контроля степени загрузки конвейера / Method of Loading Efficiency Control. Технология SENUMAC: способ автоматического управления загрузкой поточно-

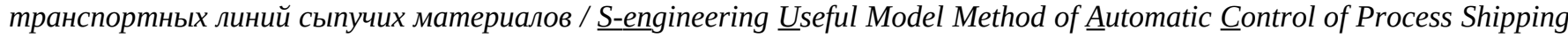
line upload of granular materials.

Разработка таких систем включает несколько этапов, одним из которых является этап исследования функционирования ПТЛ, изучение их свойств как объектов управления с целью дальнейшего отражения этих свойств в алгоритмах управления. Особое внимание уделяется изучению подсилосных задвижек, так как их конструкция, размеры, принцип установки значительно влияют на процесс истечения зерна.

В данной статье рассматривается экспериментальное получение математических моделей истечения зерна из подсилосных задвижек для дальнейшей их реализации в алгоритмах управления АСОЗ ПТЛ перегрузки зерна на суда.

Abstract: S-engineering company, which is part of the holding company SE Group International, occupies a leading 